\title{
Fatigue life prediction of textile/woven hybrid composites
}

\begin{abstract}
The topic of fatigue and life prediction of hybrid composite structures is normally complicated and still limited. The aim of this chapter is to provide an overview of the fatigue life prediction for individual and hybrid woven fiber composite materials and highlights the most effective factors on their fatigue strength by recalling the previous methods, the main reasons for the accelerating popularity of composites in weight critical applications, describing the current status, and attempting to draw recommendations for future research.
\end{abstract}

Keywords: Fatigue life prediction; Hybrid composites; Mechanical properties; Natural fibers; Polymer composites; Woven fiber 